Ensayo científico

Los textos publicados son responsabilidad exclusiva de sus autores

\title{
Metodologías para el estudio de figuras históricas en educación
}

\section{Methodologies for the study of historical figures in education}

Recibido 18/10/2021

\author{
Elba Margarita Berríos Castillo \\ Universidad de El Salvador \\ elba.berrios@ues.edu.sv \\ https://orcid.org/0000-0002-3035-2029
}

Aceptado 16/11/2021

\section{Referencia}

Berríos Castillo, E. M. (2021). Metodologías para el estudio de figuras históricas en educación. Revista Guatemalteca de Educación Superior, 5(1), 102-117.

https://doi.org/10.46954/revistages.v5i1.78

\section{Resumen}

OBJETIVO: analizar las principales metodologías que se aplican para el estudio de figuras históricas en educación. MÉTODO: la metodología utilizada es el análisis documental que permite indagar las diferentes investigaciones histórico-pedagógicas en el contexto latinoamericano. Los países seleccionados son Cuba, México, Argentina, Perú, Chile, Colombia, Honduras, Costa Rica, República Dominicana y El Salvador. RESULTADOS: se logró identificar en las diferentes investigaciones los pasos metodológicos que sustentan el estudio de una figura histórica, su trascendencia por sus aportes a las ciencias de la educación o pedagógicas, a la investigación y las prácticas educativas, en el contexto y el tiempo que le tocó vivir. CONCLUSIÓN: el estudio de las personalidades históricas, como parte fundamental de la 
historia de la pedagogía latinoamericana presenta limitaciones por la carencia de procedimientos metodológicos que unifiquen y proporcionen mayor coherencia y sistematicidad al mismo. Cuba es uno de los países que más ha trabajado en la construcción de una herramienta metodológica, lo cual permite la identificación, la divulgación de la vida y obra de grandes figuras de alcance nacional e internacional. Los autores estudiados coinciden con el enfoque historiográfico aplicado en sus investigaciones en cambio los investigadores cubanos consideran el enfoque histórico-lógico.

\section{Abstract}

OBJECTIVE: to analyze the main methodologies applied to the study of historical figures in education. METHOD: the methodology used is the documentary analysis that allows to investigate the different historical-pedagogical investigations in the Latin American context. The selected countries are Cuba, Mexico, Argentina, Peru, Chile, Colombia, Honduras, Costa Rica, the Dominican Republic and El Salvador. RESULTS: it was possible to identify in the different investigations the methodological steps that support the study of a historical figure, its significance due to its contributions to educational or pedagogical sciences, research and educational practices, in the context and time that he had to live. CONCLUSION: the study of historical personalities, as a fundamental part of the history of Latin American pedagogy, presents limitations due to the lack of methodological procedures that unify and provide greater coherence and systematicity to it. Cuba is one of the countries that has worked the most in the construction of a methodological tool, which allows the identification and dissemination of the life and work of great figures of national and international scope. The authors studied coincide with the historiographic approach applied in their research, whereas Cuban researchers consider the historical-logical approach.

\section{Palabras clave:}

figura o personalidad histórica, metodología, investigaciones histórico-pedagógicas.

\section{Keywords:}

historical figure or personality, methodology, historicalpedagogical research. 


\section{Introducción}

La historiografía salvadoreña ha estado marcada, en gran medida, por un enfoque descriptivo, de ahí que, el reto de los investigadores es de ir a la búsqueda de las raíces. desde una concepción materialista de la historia, es decir abordar científicamente el decursar histórico. Asimismo, la historia de la educación y del pensamiento pedagógico como parte de la historia de El Salvador, no se sustrae de esta problemática y retos, por lo que se debe profundizar en las raíces del saber pedagógico y educativo y en su valor patrimonial para la nación y el mundo.

Aunque existen valiosos trabajos que abordan el quehacer educacional, teórico y práctico, aún son insuficientes las obras totalizadoras que revelen las regularidades del desarrollo de la educación y del pensamiento pedagógico salvadoreño y también son escasos los estudios de muchos de los hechos y figuras históricas. Estas dificultades complejizan y limitan el alcance de las investigaciones que deben acometerse, más ello no puede resultar un freno para el investigador ya que, si se pretende conformar la historia de la educación y del pensamiento pedagógico, es un requisito analizar el fenómeno en toda su multiplicidad, incorporando los hombres y mujeres que intervinieron en su acontecer.

Por ello es preciso que los estudios histórico pedagógicos aborden entre sus problemas la contribución a la educación y al pensamiento pedagógico de su tiempo de un grupo de educadores sociales y pedagogos, quienes fueron, en menor o mayor medida figuras complejas, contradictorias, en ocasiones limitadas ideológicamente, pero que al incidir con amplitud en la esfera educacional dejaron huellas en el quehacer teórico y práctico. Sólo así, retomando la realidad en su complejidad, podrá conformarse el cuadro general de la historia de la educación y del pensamiento pedagógico en El Salvador. 
Este trabajo es producto del capítulo uno de la tesis doctoral que estoy trabajando como requisito previo para recibir el título de doctora en Educación con especialidad en Educación Superior del Programa Interdisciplinario de la Universidad de El Salvador.

\section{Contenido}

\section{Metodologías para el estudio de figuras históricas en educación}

Aquí se analizan las principales metodologías para el estudio de figuras históricas en educación, para ello se indagan diferentes investigaciones histórico-pedagógicas en el contexto latinoamericano, donde existe una tradición de estudios históricos en las ciencias pedagógica y de la educación.

\section{Metodologías en el estudio de figuras históricas en educación en el contexto latinoamericano}

El análisis de diferentes metodologías en el estudio de figuras históricas en varios países de Latinoamérica se realizó con la intención, de sustentar los aspectos o pasos que no deben faltar una metodología que sustente el estudio de una figura y su trascendencia por sus aportes a las ciencias de la educación o pedagógicas, a la investigación y las prácticas educativas, en el contexto y el tiempo que le tocó vivir. Entre los países seleccionados están Cuba, México, Argentina, Perú, Chile, Colombia, Honduras, Costa Rica, República Dominicana y El Salvador. En ello se destaca trabajos dirigidos al estudio de figuras nacionales e internacionales que, con sus reflexiones teóricas y prácticas educativas, son parte de la historia de la educación en la región, lo que demuestra la necesidad de reconocer en la historia del pensamiento y la práctica educativa de hombres y mujeres su contribución al desarrollo de las naciones y sus ciudadanos. 
De Cuba se consultaron obras de autores como; Fernández (2004), Barrabia (2005), Luis (2006), Blanco (2008), Romero (2014), Mulen (2015), Sáenz (2017) y Quiñones (2017), todas las fuentes consultadas desarrollaron y sustentaron las metodologías como parte de investigaciones para obtener el grado científico de doctor en Ciencias Pedagógicas. Los investigadores cubanos, consideran en sus investigaciones el enfoque histórico-lógico, desde una posición marxista de la historia lo que significa que abordan la lógica y la historia como unidad y sirve de hilo metodológico rector en la investigación del objeto de estudio.

Las investigaciones analizadas, se desarrollan durante las primeras décadas del siglo XXI, entre las figuras que se estudian están: José Ingenieros, Jaime Torres Bodet, Simón Rodríguez, Raúl Ferrer, José Elpidio Pérez Somossa, Paulo Freire, Hugo Rafael Chávez Frías, Augusto Salazar Bondy, Enma Elvira Guerra Cardona, Rosa María Angulo Díaz-Canel, Francisco Larroyo, María Dámasa Jova Baró, José Vasconcelos, Fidel Castro Ruz, Alberto Masferrer, Oswaldo Robles lo que demuestra que los aportes a la educación y pedagogía de un país, puede analizarse desde las diferentes funciones sociales de una figura que, por sus aportes a la sociedad y formación de sus ciudadanas, trasciende en la historia.

En la investigación realizada por Fernández (2004), se persiguió como propósito determinar en la obra educativa de Raúl Ferrer las principales ideas relacionadas con la misión del maestro que constituyen una contribución a la educación cubana actual. Para ello siguió como proceder metodológico 1. Definición de conceptos sobre la categoría de análisis. Profundización teórica, 2. Acopio de documentación sobre el autor, 3. Determinación del contexto epocal en que interactúa la personalidad objeto de estudio. Establecimiento de la cronología y de una posible periodización, 4. Estudio del conjunto de su obra, 5. Elección de un o varias obras escritas, 6. Talleres de reflexión y foros de discusión, 7. Valoración de la obra escogida sobre la base de la categoría de análisis. 
En el caso de la autora Barrabia (2005) en su obra Contribución de Fidel Castro Ruz a la formación del hombre nuevo en la escuela cubana de 1959-1975 describe los elementos metodológicos para abordar el estudio de una personalidad histórica: 1. Selección del período histórico de la investigación, 2. Establecer las variables, dimensiones e indicadores para la comprensión más integral y científica de su contribución; para la autora esto constituye una guía metodológica para posteriores investigaciones.

Asimismo Luis (2006) en su estudio de la obra educativa de la maestra santaclareña María Dámasa Jova Baró (1890-1940) emplea por primera vez una metodología cienciológica la cual permite organizar, planificar y dirigir la actividad científicainvestigativa, que persigue ofrecer un estudio que integralmente muestre la interacción que se da entre lo caracterológico de la figura y la contextualización de la misma, donde se refleje cómo los aspectos económicos, sociales, histórico-lógicos, psicológicos, pedagógicos, culturales, educativos y orgánicoestructurales determinan el desarrollo de la personalidad estudiada.

De igual modo Blanco (2008) en su trabajo: Estrategia Metodológica para contribuir al mejoramiento del trabajo con las personalidades históricas en los docentes de la Educación Técnica y Profesional en Pinar del Río. Propone una estrategia metodológica para el tratamiento a las personalidades históricas, que contiene los siguientes aspectos: 1. Contexto histórico en que se desarrolla, medio geográfico, interés clasista, contexto familiar, círculo de personas que más influyeron en su vida, 2. Rasgos que caracterizan su personalidad vistos a través de su vida y obra, 3. Correlación entre su modo de pensar de una parte y las tendencias de desarrollo de su época por la otra. 4. Conclusiones respecto al papel jugado por el personaje en la historia local, nacional y/o universal, 5. Valoración del papel desempeñado por el individuo en la historia.

Igualmente, Romero (2014) utiliza para el estudio histórico la periodización como recurso metodológico en la determinación de los aportes educativos de la figura objeto de análisis; esto permite ubicarla en los planos general y particular. En la periodización que se propone se asumen períodos, que, a 
juicio del autor, posibilitan hacer un análisis general que guarda estrecho vínculo con el desarrollo de la obra pedagógica y la actividad educativa de la figura.

De la misma manera Mulen (2015) habla de un proceder metodológico para el estudio de una personalidad de la educación que en su contribución predomina el aspecto práctico, este autor asume los siguientes principios para el estudio del proceso histórico-pedagógico: 1. Principio del carácter histórico, social y clasista de la educación, 2. Principio sobre la consideración de las categorías histórico-pedagógicas, 3. Principio de la unidad entre lo filosófico y lo pedagógico, 4. Principio de la periodización del proceso histórico-pedagógico, 5. Principio de la vinculación entre la unidad y la diversidad educativa, 6. Principio del carácter procesal del fenómeno histórico-pedagógico.

De igual forma Sáenz (2017) emplea en el conocimiento del proceso histórico-pedagógico un sistema categorial que permiten ir del fenómeno a la esencia, de lo abstracto a lo concreto, ellas son: el contexto histórico-social y educativo, periodización, la personalidad de la figura estudiada, el quehacer pedagógico (aspectos teóricos y prácticos), la esencia del pensamiento pedagógico que se descompone en dimensiones y características, vigencia y legado.

Del mismo modo Quiñones (2017) plantea un proceso metodológico considerando los siguientes aspectos: 1. Evolución histórica del concepto de educador social, dentro de este se hace un análisis de los estudios sobre educadores sociales y se analiza el educador social en el contexto socio educativo del país, 2. El contexto histórico-social en que vivió y se formó la figura, aquí también se establece una periodización de la evolución histórica del pensamiento político-educativo de la personalidad, 3. Los rasgos que caracterizan a la figura como educador social, 4. La contribución de la personalidad a la educación, 5. La vigencia de su pensamiento educativo. 
El análisis de las anteriores investigaciones, condujo a constatar que, la metodología utilizada en ellas se caracteriza por permitir un acercamiento a las personalidades históricas de forma integral, lo que significa conocer todo el despliegue de las actividades de la figura en el tiempo y el espacio se aplica con flexibilidad sobre la base de un enfoque dialéctico. De estas metodologías, en la presente investigación se asume el enfoque histórico-lógico sustentado por los autores cubanos y además algunos elementos de la metodología aplicada por ellos como es el sistema categorial: contexto socio-histórico y educativo, periodización, la personalidad de la figura estudiada, el quehacer pedagógico (aspectos teóricos y prácticos), la contribución del pensamiento pedagógico que se descompone en variables, dimensiones e indicadores, vigencia y trascendencia.

Las investigaciones analizadas en México, están dirigidas a sustentar la actividad educativa de muchas figuras; así se tienen autores como; Ávila (1988), Segovia (2003), Aguayo (2006), Guillén (2008), Corona (2009) y Razo (2010). Los investigadores estudiados asumen que toda investigación que pretende consignar el pensamiento pedagógico y la obra educativa de los grandes pensadores mexicanos, tiene que recurrir necesariamente a la revisión histórica ya que con ello adquiere contexto y significado el análisis pedagógico. Los autores mexicanos aplican en sus investigaciones el enfoque historiográfico, según ellos, como las diferentes visiones de abordar el estudio, la escritura e interpretaciones del pasado.

En el caso de Ávila (1988) en su estudio: Fundamentos filosóficos del pensamiento antropológico y pedagógico de Paulo Freire. Establece una metodología que él llama estrategia la cual tiene como punto de partida describir los momentos relevantes del personaje, como segundo elemento aborda la base filosófica del pensamiento pedagógico y por último el carácter filosófico de la antropología de la figura. Por su parte Segovia (2003) desarrolla su investigación: La influencia del Pensamiento Pedagógico del Dr. Francisco Larroyo en el diseño del plan de estudios de Pedagogía vigente en la Facultad de Filosofía y Letras en la UNAM. México, desde el siguiente proceso metodológico. 1. Un esbozo histórico este se refiere al contexto 
político, social, económico, cultural y educativo en el cual se desarrolló el personaje, 2. La vida y obra de la figura, 3. El pensamiento filosófico y pedagógico y 4 . El objeto de estudio de la investigación; según el autor este enfoque metodológico permite comprender y organizar el fenómeno educativo a través de un proceso sistemático, considerando las dos dimensiones la individual y la social.

El abordaje metodológico que propone Aguayo (2006) es el siguiente: 1. La vida de la personalidad que abarca su educación, sus intereses intelectuales, descripción de su persona y su formación, actividades públicas, influencias académicas y su bibliografía, 2. Una periodización que permite dividir en etapas la trayectoria de vida de la figura histórica. Para este autor Guillén (2008) la revisión histórica de los hechos educativos, adquiere relevancia en la medida en que permite integrar la experiencia educativa con la comprensión del fenómeno objeto de estudio y enriquecer la explicación del mismo. El abordaje metodológico que este hace en su investigación se detalla a continuación: 1. Marco histórico para ubicar la obra educativa y el pensamiento pedagógico de la figura, 2. Semblanza biográfica del personaje, 3. Obra educativa de la figura, 4. Pensamiento pedagógico de la personalidad estudiada.

El abordaje metodológico según la autora Corona (2009) consiste en una sistematización haciendo uso de la periodización, así se tiene: 1. Los primeros años del personaje: Antecedentes familiares, su niñez, la adolescencia y su juventud, 2. Primer período, 3. Segundo período, 4. Contribución educativa de la figura estudiada. En su investigación Razo (2010) plantea que por tener un carácter histórico la metodología propuesta para el estudio de la personalidad debe tener como punto de partida el análisis del contexto histórico, político, social, religioso y educativo en donde se desarrolló la vida de la figura seguidamente están los datos biográficos, obras y conceptos claves del pensamiento del personaje y por último la práctica de la personalidad. 
La revisión de estos estudios permitió reconocer que, la metodología aplicada se caracteriza por tener como punto de partida un marco o contexto histórico, luego se analiza la vida y obra del pensador estableciendo una periodización, el análisis está dirigido esencialmente al estudio de los aportes del personaje a la educación mexicana. De acuerdo al enfoque historiográfico sustentado por los autores mexicanos son mínimos los elementos que pueden tributar a la presente investigación, en este caso estaría retomando la categoría de la periodización.

Entre los investigadores suramericanos, en Argentina se tienen: Amuchástegui (2008), Durán, (2015); en Colombia: García (2010), Márquez (2016); en Chile: Reyes (1995) y en Perú: Mendoza (2008). El enfoque metodológico según este autor Amuchástegui (2008) está constituido por dos secciones: la primera está dirigida al análisis de la dimensión interna y contiene el espacio, las perspectivas teóricas y las proyecciones temáticas; la segunda sección se refiere al análisis de la dimensión externa, esto permite tener una mayor amplitud al estudio de la obra de la figura.

Asimismo, Durán, (2015) organiza una metodología estructurada en tres partes: la primera está relacionada con la unidad del pensamiento de la figura, la segunda las influencias filosóficas del pensamiento de juventud e independencia del pensamiento de madurez del personaje y la tercera la originalidad del pensamiento de madurez de la personalidad. De la misma manera García (2010) dice que la metodología para realizar estudios de figuras históricas tiene que tener como punto de partida la experiencia de la personalidad; luego sus propuestas y las categorías que sustentan su obra.

Igualmente, Márquez (2016) aborda un camino metodológico así le llama ella para el estudio de una personalidad con un enfoque histórico-hermenéutico y comprende los siguientes pasos: El contexto, la teoría y el análisis con esto se logra sistematizar la vida y obra de una figura histórica. La metodología que utiliza Reyes (1995) para el estudio de una figura es la siguiente: 1. Antecedentes biográficos, 2. Aspectos filosóficos del pensamiento y 3 . Aportes de la personalidad; esto permite la sistematización de la vida y obra de una personalidad. 
En el caso de Mendoza (2008) sugiere una metodología para el estudio de figuras históricas: 1. Vida, obras y evolución del pensamiento del personaje, 2. Tesis propuestas por la personalidad, 3. Vigencia de su pensamiento. Los autores estudiados coinciden en que sus trabajos son investigaciones cualitativas, pero no tienen claridad con el enfoque aplicado excepto el estudio colombiano de la autora (Márquez, 2016) que asume el enfoque histórico-hermenéutico.

En Centro América, se analizaron metodologías sustentadas por investigadores en el área de la historia de la Pedagogía, en Costa Rica: García (2005) y en Honduras: Alonso (2006). Así se tiene que García (2005) propone una metodología partiendo de la posición teórica y crítica de la figura histórica seguido de los aportes del pensamiento de la personalidad terminando en la vigencia y actualidad de sus planteamientos. El enfoque metodológico planteado por este autor Alonzo (2006) es el siguiente: 1. El contexto socio-político de la obra de la figura, 2. La propuesta pedagógica del pensador y 3. Las obras de la personalidad para poder elaborar las tipologías que permitan explicar el fenómeno en investigación.

Se constató en estas investigaciones la no existencia de una metodología que tenga como propósito la contribución del pensamiento pedagógico de una figura histórica a la educación superior. En El Salvador, al igual que los otros países de Centro América la producción de tesis de figuras históricas en educación es escasa, así se tienen autores como: La metodología propuesta en Aparicio (2007) para estudiar una figura es la siguiente: 1. El entorno histórico y político del personaje, 2. El perfil de la personalidad, 3. Establecimiento de categorías para el análisis de su obra. Esto permite profundizar en la vida y obra del pensador en investigación.

En el caso de Carranza et al. (2015) establecen un procedimiento sistemático cualitativo como metodología para el estudio de la figura histórica la cual permite relacionar una situación con un contexto particular esto genera el entendimiento de un fenómeno educativo, psicológico, comunicativo o cualquier otro 
que sea concreto. El proceder metodológico fue el siguiente: 1. Las obras escritas por la personalidad, 2. Las corrientes filosóficas que influyeron en el pensamiento de esta. 3. Establecimiento de categorías para el análisis de la figura.

La metodología que optó Parada (2016) en su investigación fue guiada bajo el método histórico convirtiéndose la biografía y la periodización como herramientas claves para conocer la actuación del personaje en cada una de las ramas y períodos en que se desempeñó. Los autores estudiados a nivel nacional comparten que el enfoque que predomina es el cualitativo y el método que ha guiado las investigaciones es el histórico, al mismo tiempo se han auxiliado de la biografía y de la periodización.

\section{Conclusiones}

Al hacer una revisión bibliográfica de las diferentes investigaciones histórico-pedagógicas realizadas en América Latina con el objetivo de identificar la metodología utilizada para el estudio de figuras en educación, se puede establecer que el estudio de las personalidades históricas, como parte fundamental de la historia de la pedagogía latinoamericana presenta limitaciones por la carencia de procedimientos metodológicos que unifiquen y proporcionen mayor coherencia y sistematicidad al mismo.

No obstante, Cuba es uno de los países que más ha trabajado en la construcción de una herramienta metodológica para la realización de investigaciones históricas, lo cual permite la identificación, la divulgación de la vida y obra de grandes figuras de alcance nacional e internacional. Los autores estudiados coinciden con el enfoque historiográfico aplicado en sus investigaciones en cambio los investigadores cubanos consideran el enfoque histórico-lógico. 


\section{Referencias bibliográficas}

Aguayo, E. I. (2006). El Pensamiento Filosófico - Psicológico de Oswaldo Robles. México D.F.: Universidad Nacional Autónoma de México. https://repositorio.unam.mx/ contenidos/75632

Alonzo, G. I. (2006). La relación horizontal en el pensamiento pedagógico de Paulo Freire. Tegucigalpa, Honduras: Universidad Pedagógica Nacional Francisco Morazán. http://www.cervantesvirtual.com/nd/ark:/59851/ bmc931f7

Amuchástegui, R. H. (2008). Michel Foucault y la Visoespacialidad Análisis y Derivaciones. Buenos Aires, Argentina: Universidad Buenos Aires. https://www. scribd.com/doc/78918213/Amuchastegui-RodrigoHugo-Tesis-de-Doctorado-Michel-Foucault-y-lavisoespacialidad

Aparicio, L. A. (2007). Alberto Masferrer: Pedagogo Político. San Salvador: Universidad Pedagógica de El Salvador. ISBN 978-99923-30-07-4 https://issuu. com/bibliotecapedagogica/docs/alberto_masferrer_ pedagogo_pol_tic

Ávila, A. (1988). Tesis de Licenciatura. Fundamentos filosóficos del pensamiento antropológico y pedagógico de Paulo Freire. México D. F., México. https://repositorio.unam. $\mathrm{mx} /$ contenidos/217832

Barrabia, O. (2005). Tesis Doctoral Contribución de Fidel Castro Ruz a la formación del hombre nuevo en la escuela cubana de 1959-1975. CD-ROM. La Habana. Cuba: ISPEJV.

Blanco, O. (2008). Tesis de Maestría. Estrategia Metodológica para contribuir al mejoramiento del trabajo con las personalidades históricas en los docentes de la Educación Técnica y Profesional en Pinar del Río. Pinar del Río. CD-ROM. Pinar del Río, República de Cuba. 
Carranza, R. E., Rivas, O. A., \& Alvarenga, B. I. (2015). Vigencia del pensamiento pedagógico de José Ingenieros. San Salvador, El Salvador: Universitaria. Tesis Vigencia Del Pensamiento Pedagógico de José Ingenieros - Julio 2015 PDF I PDF I Moralidad I Teorías filosóficas (scribd. com)

Corona, M. d. (2009). Tesis Maestría. Un estudio HistóricoPedagógico del Pensamiento y obra educativa de don Jaime Torres Bodet. México D.F.: Dirección General de Bibliotecas UNAM. https://repositorio.unam.mx/ contenidos/260315

Durán , M. (2015). Tesis Doctoral. El concepto de educación popular en el pensamiento de Simón Rodríguez. Buenos Aires, Argentina. http://catalogo.filo.uba.ar/cgibin/koha/opac-detail.pl?biblionumber=163549

Fernández, E. M. (2004). Tesis Doctoral. Ideas acerca de la misión del maestro en la obra educativa de Raúl Ferrer. Su contribución a la educación cubana. CD-ROM Villa Clara, República de Cuba.

García, B. Y. (2010). Pensamiento de Simón Rodríguez: La educación como proyecto de inclusión social. Revista Colombiana de Educación, 134-147. Redalyc. Pensamiento de Simón Rodríguez: la educación como proyecto de inclusión social

García, J. (2005). El Pensamiento Pedagógico de José Martí acerca de la Formación docente y el Curriculum Educativo. Revista Educación, Universidad de Costa Rica. https://www.redalyc.org/pdf/440/44029205.pdf

Guillén, B. G. (2008). Obra Educativa y pensamiento Pedagógico de José Vasconcelos. México, D.F.: Dirección General de Bibliotecas de la UNAM. https:// repositorio.unam.mx/contenidos/84473

Luis, N. (2006). Estudio de la obra educativa de la maestra santaclareña María Dámasa Jova Baró (1890-1940). Santa Clara, Cuba: Editorial Universitaria del Ministerio de Educación Superior. Nancy Luis.pdf (uclv.edu.cu) 
Márquez, M. L. (2016). Tesis Doctoral. Los relatos de nación en Venezuela como escenario de construcción y de disputa de la hegemonía del gobierno de Huo Chávez (1999-2012). Pontificia Universidad Javeriana. Bogotá, Colombia. https://1library.co/document/4yr3xk7yrelatos-nacion-venezuela-escenario-construccionhegemonia-gobierno-chavez.html

Mendoza, A. J. (2008). La Filosofía de la educación de Augusto Salazar Bondy. Lima, Perú: Universidad Nacional Mayor de San Marcos. https://eltalondeaquiles.pucp.edu.pe/ wp-content/uploads/2017/10/mendoza_ra.pdf

Mulen, A. (2015). Enma Elvira Guerra Cardona. Estudio de su obra Educativa en Guantánamo. Santiago de Cuba: Universidad de Oriente. http://www.eduniv.cu/items/ show/39233

Parada, S. B. (2016). Fabio Castillo Figueroa y sus períodos rectorales: 1963-1966/1990-1995. San Salvador, El Salvador, C. A.: Universitaria. https://ri.ues.edu.sv/id/ eprint/12414/1/14102846.pdf

Quiñones, Á. E. (2017). Contribución de Hugo Rafael Chávez Frías como Educador Social a la Educación Venezolana. Caracas, Venezuela: Instituto Pedagógico Latinoamericano y Caribeño. CD-ROM

Razo, T. (2010). La Educación Femenina Religiosa en el Pensamiento Pedagógico y la Práctica Educativa de San Jerónimo (340 - 420). México D.F.: Universidad Nacional Autónoma de México. https://repositorio.unam.mx/ contenidos/429172

Reyes, J. (1995). Tesis de Licenciatura. En torno a Paulo Freire. Santiago, Chile. https://servicioskoinonia.org/biblioteca/ general/TesisFreire.pdf

Romero, J. (2014). Tesis Doctoral. Contribución de la obra pedagógica de la doctora Rosa María Angulo DíazCanel a la enseñanza de la Biología en Cuba entre 1954 a 1983. CD-ROM, La Habana, República de Cuba. 
Sáenz, F. G. (2017). Tesis Doctoral. Desarrollo del Pensamiento Pedagógico de Don Bosco: Vigencia y Legado. CDROM. Ciudad de La Habana, Cuba: CEPES.

Segovia, S. (2003). Tesis de Licenciatura. La influencia del Pensamiento Pedagógico del Dr. Francisco Larroyo en el diseño del plan de estudios de Pedagogía vigente en la Facultad de Filosofía y Letras en la UNAM. México D.F.: Dirección General de Bibliotecas UNAM. https:// repositorio.unam.mx/contenidos/440867

\section{Sobre la autora}

La autora desde 1992 es profesora a tiempo completo de la carrera de Medicina de la Facultad Multidisciplinaria Oriental, Universidad de El Salvador. Estudió Sociología con maestría en Profesionalización de la Docencia Superior y maestría en Métodos y Técnicas de Investigación Social, candidata a doctora en Educación con la especialidad en Educación Superior del programa Interdisciplinario de la Universidad de El Salvador mediante convenio con las Universidades de La Habana y Ciencias Pedagógicas "Enrique José Varona" de la República de Cuba.

\section{Conflicto de intereses}

Declara no tener ningún conflicto de intereses.

\section{Declaración de consentimiento informado}

El estudio se realizó con fines educativos, respetando el Código de ética y buenas prácticas editoriales de publicación.

\footnotetext{
Derechos de uso Copyright@ 2021 por Elba Margarita Berríos Castillo BY

Este texto está protegido por la Licencia Creative Commons Atribución 4.0 Internacional.

Usted es libre para compartir, copiar y redistribuir el material en cualquier medio o formato y adaptar el documento, remezclar, transformar y crear a partir del material para cualquier propósito, incluso comercialmente, siempre que cumpla la condición de atribución: usted debe reconocer el crédito de una obra de manera adecuada, proporcionar un enlace a la licencia, e indicar si se han realizado cambios. Puede hacerlo en cualquier forma razonable, pero no de forma tal que sugiera que tiene el apoyo del licenciante o lo recibe por el uso que hace.
} 